IRA-International Journal of Applied Sciences ISSN 2455-4499; Vol.05, Issue 03 (2016)

Institute of Research Advances

Pg. no. 110-114

http://research-advances.org/index.php/IRAJAS

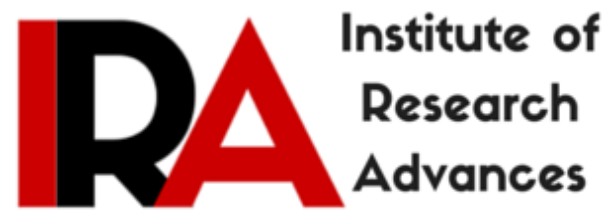

\title{
Oxidation of Benzaldehyde and Substituted Benzaldehydes by Permanganate under Phase Transfer Catalysis in Non Polar Solvents
}

\section{K. Bijudas and P. Bashpa}

Department of Chemistry, N. S. S. College, Manjeri, Malappuram, Kerala, 676122, India.

Type of Review: Peer Reviewed.

DOI: http://dx.doi.org/10.21013/jas.v5.n3.p1

\section{How to cite this paper:}

Bijudas, K., \& Bashpa, P. (2016). Oxidation of Benzaldehyde and Substituted Benzaldehydes by Permanganate under Phase Transfer Catalysis in Non Polar Solvents. IRA-International Journal of Applied Sciences (ISSN 2455-4499), 5(3), 110-114. doi:http://dx.doi.org/10.21013/jas.v5.n3.p1

(C) Institute of Research Advances

\section{(c) EY-NC}

This work is licensed under a Creative Commons Attribution-Non Commercial 4.0 International License subject to proper citation to the publication source of the work.

Disclaimer: The scholarly papers as reviewed and published by the Institute of Research Advances (IRA) are the views and opinions of their respective authors and are not the views or opinions of the IRA. The IRA disclaims of any harm or loss caused due to the published content to any party. 


\section{ABSTRACT}

Phase transfer catalysed oxidation of benzaldehyde and substituted benzaldehydes by permanganate ion have been studied in non polar solvents like ethyl acetate and toluene. The obtained products were charecterised by melting point determination and infra red spectral analysis. Benzoic acid and corresponding substituted benzoic acids were formed as the product with very high yield. The oxidation reactions were carried out by using various quaternary ammonium and phosphonium salts as phase transfer catalyst. The effect of non polar solvents and various phase transfer catalysts on yield of product was also carried out.
\end{abstract}

Keywords: Benzaldehyde, substituted benzaldehydes, permanganate, phase transfer catalysis, phase transfer catalyst.

\title{
INTRODUCTION
}

Oxidation reactions using suitable oxidants are very important in synthetic organic chemistry and is employed for the synthesis of various organic compounds [1-2]. Phase transfer catalysis (PTC) is relatively a new technique to carry out various reactions with high yield and under mild conditions [3-5]. Phase transfer catalysts (PT catalysts) are able to transfer the inorganic oxidant from aqueous phase to organic phase and the reaction takes place smoothly in the organic phase. Cost reduction and pollution prevention which are the driving force of a chemical industry today can be achieved by implementing PTC technique. There are reports on the synthesis of organic compounds by oxidation reactions using permanganate, chromate, hypochlorite etc. under PTC [6-10]. Kinetic studies of such type of oxidations using various oxidizing agents were also reported [11-16].

We have carried out the oxidation of benzaldehyde and substituted benzaldehydes using various quaternary ammonium and phosphonium salts as PT catalysts in non polar solvents like ethyl acetate and toluene by permanganate ion. The substituted benzaldehydes used were 4hydroxybenzaldehyde, 3-hydroxybenzaldehyde, 4-chlorobenzaldehyde, 3-chlorobenzaldehyde, 4methoxybenzaldehyde, 3-methoxybenzaldehyde, 4-nitrobenzaldehyde, 3-nitrobenzaldehyde and 4methylbenzaldehyde. The PT catalysts used were tetrabutylammonium bromide (TBAB), tetrabutylphosphonium bromide (TBPB), tetrabutylammonium hydrogen sulphate (TBAHS) and cetyltrimethylammonium bromide (CTMAB)

\section{EXPERIMENTAL SECTION}

Analar grade potassium permanganate (Merck, India) was used in the entire work. Benzaldehyde (AR, Spectrochem, India) and the substituted benzaldehydes employed in the present work namely, 4-hydroxybenzaldehyde, 3-hydroxybenzaldehyde, 4-chlorobenzaldehyde, 3chlorobenzaldehyde, 4-methoxybenzaldehyde, 3-methoxybenzaldehyde, 4-nitrobenzaldehyde, 3nitrobenzaldehyde and 4-methylbenzaldehyde (Spectrochem, India Ltd. Mumbai and Merck KGaA, Germany) were used as such. Tetrabutylammonium bromide (TBAB), tetrabutylammonium hydrogen sulphate (TBAHS) and cetyltrimethylammonium bromide (CTMAB) (Spectrochem India Ltd. Mumbai) and tetrbutylphosphonium bromide (Merck KGaA, Germany) were used as PT catalyst. The non polar solvents toluene and ethyl acetate were purified according to the standard procedure [17-18]. The purified solvents were refluxed for 1-2 hours with a mixture of PT catalyst and potassium permanganate and then distilled.

Synthetic experiments were carried out under heterogeneous condition. Benzaldehyde and substituted benzaldehydes $(0.1 \mathrm{~mol})$ dissolved in $50 \mathrm{~mL}$ of toluene or ethyl acetate which contains $0.01 \mathrm{~mol}$ PT catalyst was mixed with $50 \mathrm{~mL}$ potassium permanganate $\quad(0.5 \mathrm{~mol})$. The reaction mixture was kept at room temperature with mild stirring using a magnetic stirrer for about thirty minute. The organic layer was extracted with ether three times and the obtained organic layer was again extracted with $10 \%$ sodium bicarbonate. Both the organic and aqueous layers were separated and the aqueous layer was acidified with concentrated $\mathrm{HCl}$. The white crystalline precipitate formed was filtered, dried and weighed. The recrystallised product was analysed by melting point determination and infra red spectral technique. 


\section{RESULTS AND DISCUSSION}

Stoichiometry of the oxidation was established by equilibrating known excess concentration of permanganate with known amount of benzaldehyde under PTC condition. It was found that three moles of benzaldehyde reacts with two moles of permanganate.

$$
3 \mathrm{PhCHO}^{2} 2 \mathrm{QMnO}_{4} \longrightarrow 3 \mathrm{PhCOOH}+2 \mathrm{QOH}+2 \mathrm{MnO}_{2}+2 \mathrm{H}_{2} \mathrm{O}
$$

The product of oxidation under PTC condition was ascertained to be benzoic acid and corresponding substituted benzoic acids and the yield was found to be more than $90 \%$. The recrystallised samples of products of oxidation were characterized by its melting point and are given in table 1 . The obtained results of melting points were compared with the values of authentic samples and found to be similar. This showed that the product obtained may be benzoic acid and corresponding substituted benzoic acids. This was further confirmed by IR and UV- Visible spectral analysis of the product.

Table 1. Melting point of products on oxidation of benzaldehyde and substituted benzaldehydes

$\begin{array}{ll}\text { Sl. } & \text { Substrate } \quad \text { Melting point of product }\left({ }^{0} \mathrm{C}\right) \\ \text { No. } & \end{array}$

\begin{tabular}{lll}
\hline 1 & Benzaldehyde & $117 \pm 2$ \\
2 & 4-chlorobenzaldehyde & $241 \pm 2$ \\
3 & 3-chlorobenzaldehyde & $156 \pm 2$ \\
4 & 4-nitrobenzaldehyde & $326 \pm 2$ \\
5 & 3-nitrobenzaldehyde & $141 \pm 2$ \\
6 & 4-hydroxybenzaldehyde & $214 \pm 2$ \\
7 & 3-hydroxybenzaldehyde & $203 \pm 2$ \\
8 & 4-methylbenzaldehyde & $177 \pm 2$ \\
9 & 4-methoxybenzaldehyde & $180 \pm 2$ \\
10 & 3-methoxybenzaldehyde & $170 \pm 2$ \\
\hline
\end{tabular}

The spectral analyses of the product were carried out by taking benzaldehyde as the typical substrate. The infra red absorption spectrum was recorded from $\mathrm{KBr}$ pellets using Jasco FT-IR 4100 spectrophotometer (Japan) and is shown in Figure 1. 


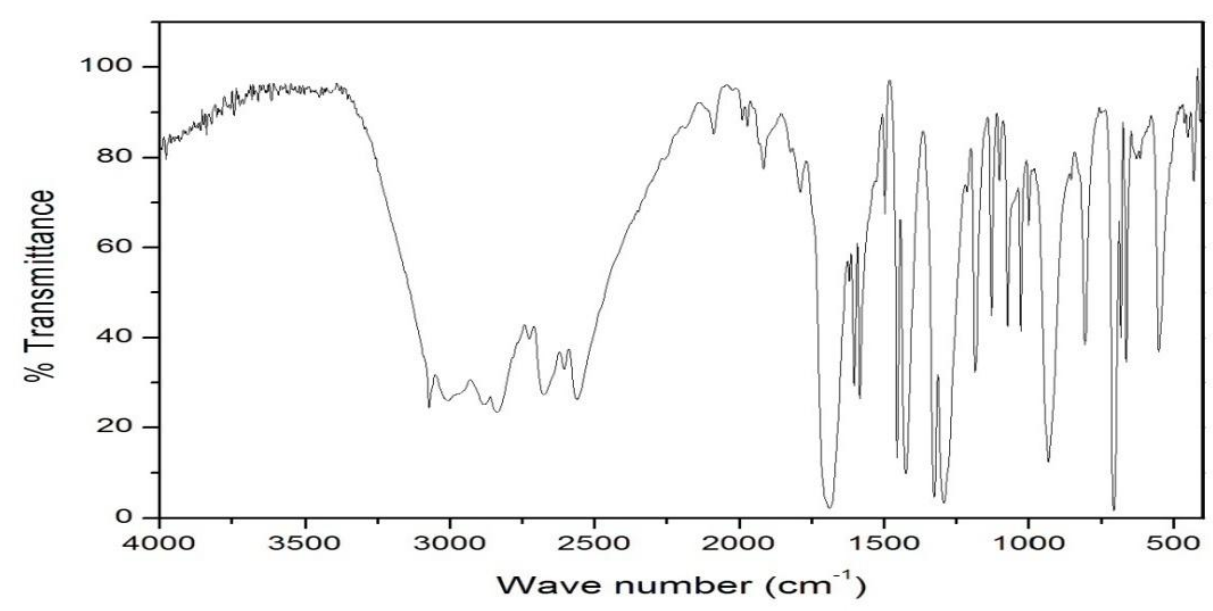

Figure 1: IR spectrum of obtained product

The infra red spectrum of the product showed sharp peaks at $3100 \mathrm{~cm}-1(\mathrm{O}-\mathrm{H}$ stretching), $1700 \mathrm{~cm}-1(\mathrm{C}=\mathrm{O}$ stretching $)$ and $1620 \mathrm{~cm}-1(\mathrm{C}=\mathrm{C})$ stretching $)$. Presence of these peaks leads to the conclusion that the product formed may be benzoic acid. Moreover, this spectrum on comparison with the infra red spectrum of pure benzoic acid showed excellent similarities.

All the above analyses, viz., melting point determination and infra red spectral studies proved the formation of benzoic acid and substituted benzoic acids by permanganate under PTC condition.

All the four PT catalysts catalyse the oxidation reactions of benaldehydes by permanganate under PTC condition. But the yield of products and ease of reaction were found to be in the order with TBPB > TBAB > TBAHS > CTMAB. This may be due to the changes in combination of alkyl groups or may be due to the difference in the activity of anions for phase transfer.

The reaction gives fairly good results in both the solvents employed, namely; ethyl acetate and toluene. The yield of benzoic acids is found to be more in ethyl acetate than that in toluene. As the polarity of ethyl acetate is higher than that of toluene it may help in increase of solubility and partitioning of quaternary salts in the organic phase which are employed as PT catalysts and this results higher reaction rate. The mechanism of the reaction can be given in Scheme 1.

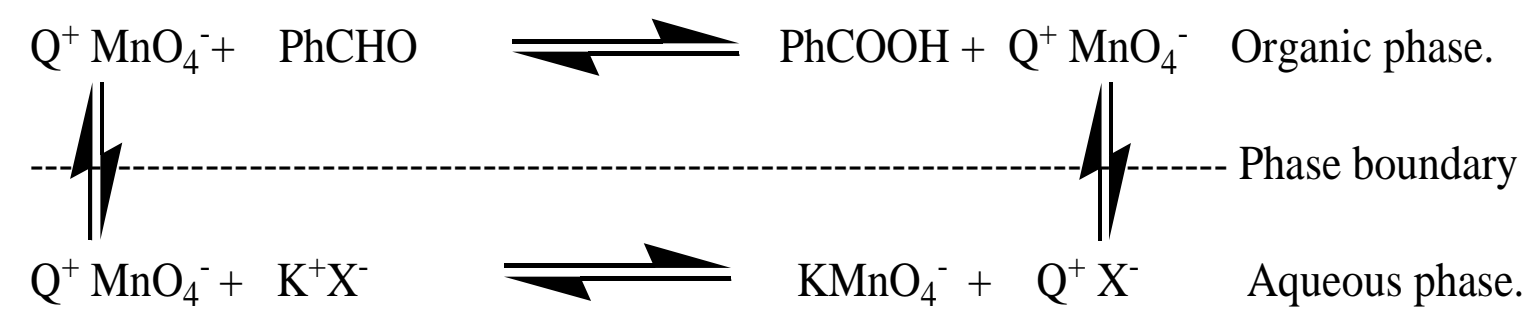

Scheme 1. PTC oxidation of benzaldehydes to corresponding benzoic acids

\section{CONCLUSION}

The PT catalysed oxidation of benzaldehyde and substituted benzaldehydes by permanganate to benzoic acid and corresponding substituted benzoic acids are found to be very smooth in non polar solvents like ethyl acetate and toluene. So such type of reactions can be employed for the oxidation of other organic substrates in non polar medium under PTC for getting desired products with high yield, less cost and in a green manner. 


\section{REFERENCES}

[1] DG Lee. Oxidation of organic compounds by permanganate ion and hexavalent chromium, Open Court: La Salle, 1980.

[2] KB Wiberg. Oxidation in organic chemistry, Academic Press, London and New York, 1965.

[3] CM Starks; C Liotta. Phase transfer catalysis, Principles and Techniques, Academic Press, New York, 1978.

[4] EV Dehmlow; SS Dehmlow. Phase transfer catalysis, VCH: Weinheim, Germany, 1993

[5] Y Sasson; R Neumann. Handbook of Phase Transfer Catalysis, Kluwer Academic Publishers; Dordrecht, 1993

[6] K Bijudas; TD Radhakrishnan Nair. Indian J. Chem., 2004, 43A, 1216-1218.

[7] K Bijudas; P Bashpa; VP Bipin; Lakshmi Nair; AP Priya; M Aswathi; C Krishnendu; $\quad$ P Lisha. Bull. Chem. React. Engg. \& Catal. 2015, 10(1), 38-42. (DOI: 10.9767/bcrec.10.1.7189.38-42).

[8] GD Yadav; CK Mistry. J. Mol. Catal. A, 2001, 172(1-2),135-149.

[9] S Abramovici; R Neumann; Y Sasson. J. Mol. Catal. A,. 1985, 29, 299-303.

[10] GA Mirafzal; AM Lozeva. Tetrahedron Lett., 1998, 39(40), 7263-7266

[11] TD Radhakrishnan Nair; P Rajendran. Indian J. Chem. 2003, 42A, 64-67.

[12] K Bijudas; P Bashpa; TD Radhakrishnan Nair. Bull. Chem. React. Engg. Catal, 2014, 9(2), 142-147.

[13] D Dey; MK Mahanti. J. Org.Chem., 1990, 55, 5848-5850.

[14] K Bijudas; TD Radhakrishnan Nair. Curr. Chem. Lett.. 2014, 3(2): 109-114. (DOI:10.5267/ j.ccl.2013.12.001

[15] MK Basheer; J Joseph; TDR Nair. Indian J. Chem., 2007, 46A, 273 - 275.

[16] D Zhao; H Ren; J Wang; Y Yang; Y Zhao. Energy Fuels. 2007, 21(5), 2543-2547. (DOI: $10.1021 / \mathrm{ef070152 \textrm {g } )}$

[17] DD Perrin; WL Armarego DR Perrin. Purification of organic compounds, Pergamon Press, Oxford, 1966.

[18] AI Vogel. Text book of practical organic chemistry, Longman, London, 1967. 